\title{
ON GENERALIZED FOURIER TRANSFORM FOR KAUP-KUPERSHMIDT TYPE EQUATIONS
}

\section{TIHOMIR VALCHEV}

Communicated by Ivaïlo M. Mladenov

Abstract. We develop the Fourier transform interpretation of the inverse scattering method for nonlinear integrable evolution equations associated with a $\mathbb{Z}_{3}$ reduced Zakharov-Shabat system for the Lie algebra $\mathfrak{s l}(3, \mathbb{C})$. A simple representative of this integrable hierarchy is the well-known Kaup-Kupershmidt equation. Our results admit a natural extention for nonlinear equations connected to a deeply reduced Zakharov-Shabat system related to an arbitrary simple Lie algebra.

\section{Introduction}

The Kaup-Kupershmidt equation (KKE) is a $1+1$ nonlinear evolution equation

$$
\partial_{t} f=\partial_{x^{5}}^{5} f+10 f \partial_{x^{3}}^{3} f+25 \partial_{x} f \partial_{x^{2}}^{2} f+20 f^{2} \partial_{x} f
$$

where $f \in C^{\infty}\left(\mathbb{R}^{2}\right)$. It is integrable by means of the inverse scattering method: it is related to a third order spectral problem [11]

$$
\left(\partial_{x^{3}}^{3}+2 f \partial_{x}+\partial_{x} f\right) y=\lambda^{3} y
$$

for some smooth function $y(x, t, \lambda)$. It can be easily transformed into a first order one [5] but related to the algebra $\mathfrak{s l}(3, \mathbb{C})$

$$
\left(\mathrm{i} \partial_{x}+q-\lambda J\right) \psi=0, \quad q=\left(\begin{array}{ccc}
u & 0 & 0 \\
0 & 0 & 0 \\
0 & 0 & -u
\end{array}\right), \quad J=\left(\begin{array}{ccc}
0 & 1 & 0 \\
0 & 0 & 1 \\
1 & 0 & 0
\end{array}\right)
$$

with some additional symmetries imposed and for an appropriately chosen new dependent variable $u$. Thus a system of the Caudrey-Beals-Coifman (CBC) type occurs. This is a typical situation when transforming a scalar differential operator of order $n$ to a $n \times n$ matrix one, see [4]. The spectral theory, the direct and inverse scattering transform for such systems have been worked out $[1-3,7]$ and more recently [9]. 
This report is to contribute to the above mentioned results on CBC systems: its main goal is to demonstrate how the generalized Fourier interpretation of the inverse scattering method (ISM) for the equations of the Kaup-Kupershmidt hierarchy can be achieved. This is a particular case of the more general problem to work out the generalized Fourier formalism for CBC systems associated with an arbitrary simple Lie algebra. In realizing our goal we are applying purely algebraic and analytic methods: "squared solutions" and recursion operator $\Lambda$ to CBC system. The "squared solutions" form a complete set and thus they generalise usual exponential functions in the Fourier analysis. The derivative operator $\mathrm{i} \partial_{x}$ to whom exponents represent eigenfunctions is to be replaced then by the $\Lambda$ operator. The integrable hierarchy of KKE can be conveniently described in terms of the $\Lambda$-operator.

\section{Some Facts From the Theory of Solitons}

We are going to briefly outline some basic concepts and results from the theory of integrable systems and introduce notation to be used further on in the text. For a more detailed introduction we refer to $[13,15]$ (see also [6]). The nonlinear evolution equations (NEE) under investigation represent the compatibility condition of two linear differential operators (Lax operators)

$$
\begin{aligned}
L(\lambda) & =\mathrm{i} \partial_{x}+U(x, t, \lambda)=\mathrm{i} \partial_{x}+q(x, t)-\lambda J \\
M(\lambda) & =\mathrm{i} \partial_{t}+V(x, t, \lambda)=\mathrm{i} \partial_{t}+\sum_{k=0}^{N} V_{k}(x, t) \lambda^{k}
\end{aligned}
$$

which holds true identically with the spectral parameter $\lambda$. The functions $q$ and $V_{k}$ take values in a complex simple Lie algebra $\mathfrak{g}$ while $J$ is chosen a real constant element of its Cartan subalgebra $\mathfrak{h} \subset \mathfrak{g}$ (for more detailed explanations on the theory of Lie algebras we refer the reader to the book [10]). We require also that the potential $q$ fulfills a zero boundary condition

$$
\lim _{x \rightarrow \pm \infty}|x|^{l} q(x, t)=0, \quad \text { for all } \quad l>0 .
$$

The interpretation of the inverse scattering method as a spectral transform is realized in several steps. The first step consists in associating to a NEE the spectral problem $L(\lambda) \psi=0$ which in our case (see (2)) is called generalized ZakharovShabat system. Its fundamental sets of solutions ${ }^{1} \psi$ take values in the group $G$

\footnotetext{
${ }^{1}$ For simplicity we are going to refer them just fundamental solutions.
} 
corresponding to the algebra $\mathfrak{g}$. Due to the requirements imposed on $J$ and $q$ the continuous spectrum of $L$ coincides with $\mathbb{R}$ in the complex $\lambda$-plane. A special type of fundamental solutions are the so-called Jost solutions

$$
\lim _{x \rightarrow \pm \infty} \psi_{ \pm}(x, t, \lambda) \mathrm{e}^{\mathrm{i} \lambda J x}=\mathbb{1}
$$

defined only for $\lambda \in \mathbb{R}$. The transition matrix between the Jost solutions is called scattering matrix

$$
\psi_{-}(x, t, \lambda)=\psi_{+}(x, t, \lambda) T(t, \lambda), \quad \lambda \in \mathbb{R} .
$$

The quantity

$$
\mathbf{f}(\lambda)=\lim _{x \rightarrow \pm \infty} V(x, t, \lambda)
$$

bears the name dispersion law and determines the evolution of the scattering data

$$
\mathrm{i} \partial_{t} T+[\mathbf{f}(\lambda), T]=0 \quad \Rightarrow \quad T(t, \lambda)=\mathrm{e}^{\mathrm{i} \mathbf{f}(\lambda) t} T(0, \lambda) \mathrm{e}^{-\mathrm{if}(\lambda) t} .
$$

This information underlies ISM which allows solving the Cauchy problem for a NEE. From now on we shall skip the time dependence in all quantities in order to simplify our notation.

In order to construct a generalization of the usual Fourier transform one needs fundamental solutions to possess analytic properties beyond the continuous spectrum of $L$, i.e., the real axis. These can be constructed starting from the Jost solutions by using the Gauss decomposition of the scattering matrix

$$
\chi^{ \pm}(x, \lambda)=\psi_{-}(x, \lambda) S^{ \pm}(\lambda)=\psi_{+}(x, \lambda) T^{\mp}(\lambda) D^{ \pm}(\lambda)
$$

where

$$
T(\lambda)=T^{-}(\lambda) D^{+}(\lambda) \hat{S}^{+}(\lambda)=T^{+}(\lambda) D^{-}(\lambda) \hat{S}^{-}(\lambda) .
$$

Hence $\chi^{+}$and $\chi^{-}$are solutions to a local Riemann-Hilbert problem ${ }^{2}$

$$
\chi^{+}(x, \lambda)=\chi^{-}(x, \lambda) G(\lambda), \quad \lambda \in \mathbb{R} .
$$

NEEs whose Lax operators are obtained from generic ones after imposing certain algebraic consraints are of primary interest in the theory of solitons due to their potential physical applications. Formally speaking the presence of algebraic constraints can be viewed as an action of some finite group $G_{R}$ called reduction group on the set of fundamental solutions $\{\psi(x, \lambda)\}$ as given below [12]

$$
\mathcal{K}[\psi(x, \kappa(\lambda))]=\tilde{\psi}(x, \lambda) .
$$

\footnotetext{
${ }^{2}$ Strictly speaking solutions to a local Riemann-Hilbert problem are the auxiliary functions $\xi^{ \pm}(x, \lambda)=\chi^{ \pm}(x, \lambda) \mathrm{e}^{\mathrm{i} \lambda J x}$ since they satisfy the normalization condition $\lim _{\lambda \rightarrow \infty} \xi^{ \pm}(x, \lambda)=\mathbb{1}$.
} 
$\mathcal{K}$ stands for a group automorphism and $\kappa: \mathbb{C} \rightarrow \mathbb{C}$ is a conformal mapping. The requirement of $G_{R}$-invariance of the linear problem $L \psi=0$ yields to certain symmetry conditions on $U$ (and therefore on $V$ ). Let us illustrate this in the following example.

Example 1. Coxeter type reduction for $\mathfrak{s l}(r+1, \mathbb{C})$.

Impose the $\mathbb{Z}_{r+1}$ reduction condition

$$
C[\psi(x, \kappa(\lambda))] C^{-1}=\tilde{\psi}(x, \lambda)
$$

where

$$
\kappa: \lambda \rightarrow \omega \lambda, \quad \omega=\mathrm{e}^{\frac{2 \mathrm{i} \pi}{r+1}}, \quad C=\operatorname{diag}\left(1, \omega \ldots, \omega^{r-1}, \omega^{r}\right) .
$$

Thus the symmetry conditions for $U$ and $V$ read

$$
\begin{aligned}
& C U(x, \omega \lambda) C^{-1}=U(x, \lambda) \Rightarrow C q(x) C^{-1}=q(x), \quad \omega C J C^{-1}=J(8) \\
& C V(x, \omega \lambda) C^{-1}=V(x, \lambda) \Rightarrow C V_{k}(x) C^{-1}=\omega^{-k} V_{k}(x)
\end{aligned}
$$

Consequently $q(x)$ and $J$ have the form

$$
q=\sum_{k=1}^{r} q_{k} H_{k}, \quad J=\sum_{\alpha \in \mathcal{A}} E_{\alpha}
$$

where $H_{k}$ for $k=1, \ldots, r$ and $E_{\alpha}$ for any root $\alpha$ are the Cartan-Weyl generators for $\mathfrak{s t}(r+1, \mathbb{C})$ while $\mathcal{A}$ stands for the set of all admissible roots, i.e., all simple roots and the minimal one. Thus we see that unlike the nonreduced case $J$ is not a Cartan element. In order to apply the general theory one should diagonalize the matrix J. Its eigenvalues are complex numbers and this in turn makes the theory of $\mathrm{CBC}$ systems quite more complicated.

\section{NEE of the Kaup-Kupershmidt Type}

As mentioned above we are studying NEEs which belong to the integrable hierarchy of the Kaup-Kupershmidt equation. This means that they have a Lax operator $L$ in the form

$$
L(\lambda)=\mathrm{i} \partial_{x}+q(x, t)-\lambda J
$$

where $q$ and $J$ belong to the algebra $\mathfrak{s l}(3, \mathbb{C})$

$$
q=\left(\begin{array}{ccc}
u & 0 & 0 \\
0 & 0 & 0 \\
0 & 0 & -u
\end{array}\right), \quad J=\left(\begin{array}{ccc}
0 & 1 & 0 \\
0 & 0 & 1 \\
1 & 0 & 0
\end{array}\right) .
$$


It proves to be more convenient to work with a diagonal matrix $J$, i.e., with $J$ being a Cartan element. This is why we are going to use from now on the representation (gauge) given below

$$
J=\left(\begin{array}{ccc}
1 & 0 & 0 \\
0 & \omega & 0 \\
0 & 0 & \omega^{2}
\end{array}\right), \quad q=\left(\begin{array}{ccc}
0 & c u & c^{*} u \\
c^{*} u & 0 & c u \\
c u & c^{*} u & 0
\end{array}\right), \quad c=\frac{\omega-1}{3} .
$$

The matrix $C$ involved in the Coxeter reduction (see Example 1) is presented in the new basis as follows

$$
C=\left(\begin{array}{lll}
0 & 0 & 1 \\
1 & 0 & 0 \\
0 & 1 & 0
\end{array}\right)
$$

The other Lax operator is a polynomial in the spectral parameter $\lambda$

$$
M(\lambda)=\mathrm{i} \partial_{t}+\sum_{k=0}^{N} V_{k}(x, t) \lambda^{k}, \quad N \neq 3 l, \quad l>0 .
$$

All coefficients $V_{k}$ take values in $\mathfrak{s l}(3, \mathbb{C})$ and the degree $N$ of the polynomial characterises each NEE. As it was demonstrated in the example above such a Lax pair is obtained from the generic one by imposing $\mathbb{Z}_{3}$ Coxeter type reduction conditions in the form (8)-(9) with $r=2$. In particular, the Kaup-Kupershmidt equation corresponds to the choice $N=5$. The imaginery function $u$ is related to the function $f$ in (1) by the following Miura transformation

$$
f=-\mathrm{i} \partial_{x} u+\frac{1}{2} u^{2} .
$$

In order to ensure so that $u$ is imaginery it is necessary to impose another reduction

$$
\begin{aligned}
& K U^{\dagger}\left(x, \lambda^{*}\right) K^{-1}=U(x, \lambda) \Rightarrow K q^{\dagger} K^{-1}=q, \quad K J^{\dagger} K^{-1}=J \\
& K V^{\dagger}\left(x, \lambda^{*}\right) K^{-1}=V(x, \lambda) \Rightarrow K V_{k}^{\dagger}(x) K^{-1}=V_{k}(x)
\end{aligned}
$$

where

$$
K=\left(\begin{array}{ccc}
1 & 0 & 0 \\
0 & 0 & \omega^{2} \\
0 & \omega & 0
\end{array}\right)
$$

Our further considerations will remain unaffected by this $\mathbb{Z}_{2}$ reduction.

Any finite order automorphism acting in a Lie algebra $\mathfrak{g}$ introduces a grading in it. Thus due to the action of the Coxeter automorphism $\mathfrak{s l}(3, \mathbb{C})$ becomes a $\mathbb{Z}_{3}$-graded Lie algebra following the rule

$$
\mathfrak{s l}(3, \mathbb{C})=\mathfrak{g}^{0} \oplus \mathfrak{g}^{1} \oplus \mathfrak{g}^{2}, \quad \mathfrak{g}^{k}=\left\{X \in \mathfrak{s l}(3) ; C X C^{-1}=\omega^{k} X\right\} .
$$




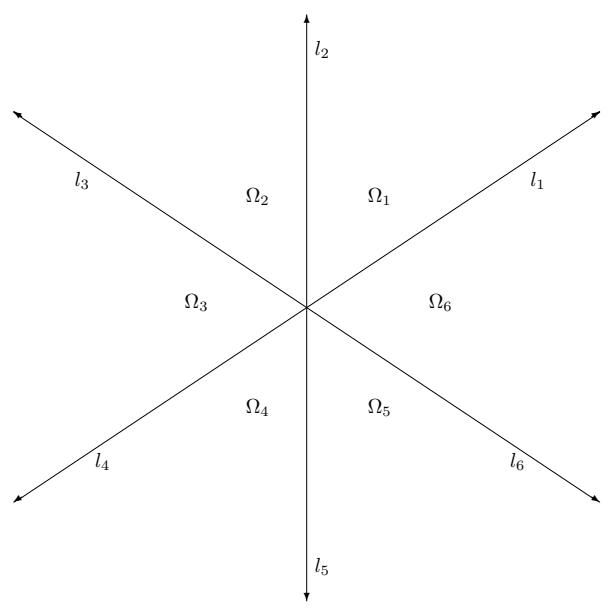

Figure 1. The continuous spectrum of $L$.

From (8) and (9) one can convince himself that

$$
q \in \mathfrak{g}^{0}, \quad J \in \mathfrak{g}^{2}, \quad V_{k} \in \mathfrak{g}^{3-k}
$$

hold true.

Since $J$ is a complex matrix (see (11)) the spectral properties of the $\mathbb{Z}_{3}$-reduced operator $L$ differ significantly from those of the nonreduced one [1-3,7]. The continuous spectrum consists of six rays $l_{a}(a=1, \ldots, 6)$ as shown on Fig.1 determined by the condition below

$$
\operatorname{Im} \lambda \alpha(J)=0 .
$$

With each ray $l_{a}$ is connected a couple of roots $\delta_{a}=\{ \pm \alpha\}$ to fulfill the requirement

$$
\delta_{a} \equiv\left\{\alpha \in \Delta ; \operatorname{Im} \lambda \alpha(J)=0, \text { for all } \lambda \in l_{a}\right\}, \quad a=1, \ldots, 6 .
$$

For $J$ chosen in the form (11) this correspondence is shown in the following table

\begin{tabular}{|c|c|c|c|}
\hline ray $l_{a}$ & $l_{1}, l_{4}$ & $l_{2}, l_{5}$ & $l_{3}, l_{6}$ \\
\hline roots of $\delta_{a}$ & $\pm\left(e_{1}-e_{2}\right)$ & $\pm\left(e_{2}-e_{3}\right)$ & $\pm\left(e_{1}-e_{3}\right)$ \\
\hline
\end{tabular}

The continuous spectrum splits the $\lambda$-plane into six sectors $\Omega_{a}$. In each sector can be introduced an ordering of the root space by specifying which roots are positive (denoted by $\Delta_{a}^{+}$) and which - negative (resp. denoted by $\Delta_{a}^{-}$) as follows

$$
\Delta_{a}^{ \pm}=\left\{\alpha \in \Delta ; \operatorname{Im}(\lambda \alpha(J)) \gtrless 0, \quad \text { for all } \quad \lambda \in \Omega_{a}\right\} .
$$


As a result each sector has its own fundamental analytic solution $\chi^{a}(x, \lambda)$. The fundamental analytic solutions of adjacent sectors are solutions to a generalised Riemann-Hilbert problem

$$
\chi^{a}(x, \lambda)=\chi^{a-1}(x, \lambda) G^{a}(\lambda), \quad \lambda \in l_{a} .
$$

The sewing functions $G^{a}(\lambda)$ can be expressed by the analogs of the Gauss factors (compare with (6))

$$
G^{a}(\lambda)=\hat{S}_{a}^{-}(\lambda) S_{a}^{+}(\lambda)=\hat{D}_{a}^{-}(\lambda) \hat{T}_{a}^{+}(\lambda) T_{a}^{-}(\lambda) D_{a}^{+}(\lambda) .
$$

It can be proven [7] that the Gauss factors read

$$
\begin{aligned}
& S_{a}^{ \pm}(\lambda)=\exp \left(\sum_{\beta \in \Delta_{a}^{+}} s_{a, \beta}^{ \pm} E_{ \pm \beta}\right), \quad D_{a}^{+}=\exp \left(\sum_{j=1}^{r} d_{a, j}^{+} H_{j}\right) \\
& T_{a}^{ \pm}(\lambda)=\exp \left(\sum_{\beta \in \Delta_{a}^{+}} t_{a, \beta}^{ \pm} E_{ \pm \beta}\right), \quad D_{a}^{-}=\exp \left(\sum_{j=1}^{r} d_{a, j}^{-} w_{0}\left(H_{j}\right)\right) .
\end{aligned}
$$

\section{Generalized Fourier Transform for Kaup-Kupershmidt Type Equations}

The role of the usual exponents in the formalism we are describing is played by the so-called squared solutions. One can construct them by using the fundamental analytic solutions of the generalised Zakharov-Shabat system

$$
\begin{aligned}
e_{\alpha}^{(a)}(x, \lambda) & =\pi\left[\chi^{a}(x, \lambda) E_{\alpha}\left(\chi^{a}(x, \lambda)\right)^{-1}\right] \\
h_{j}^{(a)}(x, \lambda) & =\pi\left[\chi^{a}(x, \lambda) H_{j}\left(\chi^{a}(x, \lambda)\right)^{-1}\right]
\end{aligned}
$$

where $\pi: \mathfrak{s l}(3) \rightarrow \mathfrak{s l}(3) / \operatorname{ker}\left(\operatorname{ad}_{J}\right)$. Next theorem holds true [14].

Theorem 2. The squared solutions form a complete system with the following completeness relations

$$
\begin{aligned}
\delta(x-y) \Pi= & \frac{1}{2 \pi} \sum_{a=1}^{6}(-1)^{a+1} \int_{l_{a}} \mathrm{~d} \lambda\left[e_{\beta_{a}}^{(a)}(x, \lambda) \otimes e_{-\beta_{a}}^{(a)}(y, \lambda)\right. \\
& \left.-e_{-\beta_{a}}^{(a-1)}(x, \lambda) \otimes e_{\beta_{a}}^{(a-1)}(y, \lambda)\right]-\mathrm{i} \sum_{a=1}^{6} \sum_{n_{a}} \operatorname{Res}_{\lambda=\lambda_{n_{a}}} G^{(a)}(x, y, \lambda) .
\end{aligned}
$$


where $\lambda_{n_{a}} \in \Omega_{a}$ are discrete eigenvalues of $L$ and

$$
G^{(a)}(x, y, \lambda)=\sum_{\alpha \in \Delta_{a}^{+}} e_{\alpha}^{(a)}(x, \lambda) \otimes e_{-\alpha}^{(a)}(y, \lambda), \quad \Pi=\sum_{a=1}^{3} \frac{E_{\beta_{a}} \wedge E_{-\beta_{a}}}{\beta_{a}(J)}
$$

Due to the existence of grading in $\mathfrak{s l}(3)$ the squared solutions and the Weyl basis have the representation

$$
e_{\alpha}=e_{\alpha, 0}+e_{\alpha, 1}+e_{\alpha, 2}, \quad E_{\alpha}=E_{\alpha, 0}+E_{\alpha, 1}+E_{\alpha, 2} .
$$

All projections above can be obtained by using "averaging" procedure with Coxeter automorphism like in the following example

$$
E_{\alpha, k}=\frac{1}{3} \sum_{l=1}^{3} \omega^{-k l} C^{l} E_{\alpha} C^{-l}, \quad k=0,1,2 .
$$

Thus one is able to write down single completeness relations also for the components of the "squared solutions"

$$
\begin{aligned}
\delta(x-y) \Pi_{k}= & \frac{1}{2 \pi} \sum_{a=1}^{6}(-1)^{a+1} \int_{l_{a}} \mathrm{~d} \lambda\left[e_{\beta_{a}, k}^{(a)}(x, \lambda) \otimes e_{-\beta_{a}, 3-k}^{(a)}(y, \lambda)\right. \\
& \left.-e_{-\beta_{a}, k}^{(a-1)}(x, \lambda) \otimes e_{\beta_{a}, 3-k}^{(a-1)}(y, \lambda)\right]-\mathrm{i} \sum_{a=1}^{6} \sum_{n_{a}} \operatorname{Res}_{\lambda=\lambda_{n_{a}}} G_{k}^{(a)}(x, y, \lambda)
\end{aligned}
$$

where

$$
G_{k}^{(a)}(x, y, \lambda)=\sum_{\alpha \in \Delta_{a}^{+}} e_{\alpha, k}^{(a)}(x, \lambda) \otimes e_{-\alpha, 3-k}^{(a)}(y, \lambda), \quad \Pi_{k}=\sum_{a=1}^{3} \frac{E_{\beta_{a}, k} \wedge E_{-\beta_{a}, 3-k}}{\beta_{a}(J)} .
$$

As a result any function $X \in \mathfrak{g}^{k}$ can be expanded over the squared solutions, namely

$$
\begin{aligned}
X= & \frac{1}{2 \pi} \sum_{a=1}^{6}(-1)^{a+1} \int_{l_{a}} \mathrm{~d} \lambda\left(X_{\beta_{a}}^{(a)} e_{\beta_{a}, k}^{(a)}-X_{-\beta_{a}}^{(a-1)} e_{-\beta_{a}, k}^{(a-1)}\right) \\
& -\mathrm{i} \sum_{a=1}^{6} \sum_{n_{a}} \sum_{\alpha \in \Delta_{a}^{+}}\left(\dot{X}_{\alpha, n_{a}}^{(a)} e_{\alpha, k, n_{a}}^{(a)}(x)+X_{\alpha, n_{a}}^{(a)} \dot{e}_{\alpha, k, n_{a}}^{(a)}(x)\right) .
\end{aligned}
$$


The components of $X$ are given respectively by the expressions

$$
\begin{aligned}
X_{\beta_{a}}^{(a)}(\lambda) & =\left[\left[e_{-\beta_{a}, 3-k}^{(a)}(y, \lambda), X(y)\right]\right], & \dot{X}_{\alpha, n_{a}}^{(a)} & =\left[\left[\dot{e}_{-\alpha, 3-k, n_{a}}^{(a)}(y), X(y)\right]\right] \\
X_{-\beta_{a}}^{(a-1)}(\lambda) & =\left[\left[e_{\beta_{a}, 3-k}^{(a-1)}(y, \lambda), X(y)\right]\right], & X_{\alpha, n_{a}}^{(a)} & =\left[\left[e_{-\alpha, 3-k, n_{a}}^{(a)}(y), X(y)\right]\right] .
\end{aligned}
$$

We have introduced above the following auxiliary notation

$$
\begin{aligned}
& e_{\alpha, k, n_{a}}^{(a)}(x)=\lim _{\lambda \rightarrow \lambda_{n_{a}}}\left(\lambda-\lambda_{n_{a}}\right) e_{\alpha, k}^{(a)}(x, \lambda) \\
& \dot{e}_{\alpha, k, n_{a}}^{(a)}(x)=\lim _{\lambda \rightarrow \lambda_{a}} \partial_{\lambda}\left(\lambda-\lambda_{n_{a}}\right) e_{\alpha, k}^{(a)}(x, \lambda) .
\end{aligned}
$$

The bracket

$$
[[X(x), Y(x)]] \equiv \int_{-\infty}^{\infty} \mathrm{d} x\langle X,[J, Y]\rangle
$$

is the so-called skew-skalar product and $\langle.,$.$\rangle stands for the Cartan-Killing form.$

Example 3. The following expansion holds true

$$
\begin{aligned}
\operatorname{ad}_{J}^{-1}\left[J^{\sigma}, q\right]= & \frac{\mathrm{i}}{2 \pi} \sum_{a=1}^{6}(-1)^{(a+1)} \beta_{a}\left(J^{\sigma}\right) \int_{l_{a}} \mathrm{~d} \lambda\left(s_{a, \beta_{a}}^{+} e_{\beta_{a}, 1-\sigma}^{(a)}+s_{a, \beta_{a}}^{-} e_{-\beta_{a}, 1-\sigma}^{(a-1)}\right) \\
& -\mathrm{i} \sum_{a=1}^{6} \sum_{n_{a}} \sum_{\alpha \in \Delta_{a}^{+}}\left(\dot{q}_{\alpha, n_{a}, 1-\sigma}^{(a)} e_{\alpha, n_{a}}^{(a)}+q_{\alpha, n_{a}}^{(a)} \dot{e}_{\alpha, n_{a}, 1-\sigma}^{(a)}\right)
\end{aligned}
$$

where $\sigma=1,2$ the coefficients are given by

$q_{\alpha, n_{a}}^{(a)}=\left[\left[e_{-\alpha, n_{a}}^{(a)}(y), \operatorname{ad}_{J}^{-1}\left[J^{\sigma}, q(y)\right]\right]\right], \quad \dot{q}_{\alpha, n_{a}}^{(a)}=\left[\left[\dot{e}_{-\alpha, n_{a}}^{(a)}(y), \operatorname{ad}_{J}^{-1}\left[J^{\sigma}, q(y)\right]\right]\right]$.

Since all indices are taken modulo 3 , i.e., the expression $1-\sigma$ equals either to 0 or 2. The expansion coefficients are derived from the Wronskian relation

$$
\left.\left(\hat{\chi}^{a} J^{\sigma} \chi^{a}-J^{\sigma}\right)\right|_{-\infty} ^{\infty}=\mathrm{i} \int_{-\infty}^{\infty} \mathrm{d} x \hat{\chi}^{a}\left[J^{\sigma}, q\right] \chi^{a}
$$

which leads immediately to

$$
\left\langle\left.\left(\hat{\chi}^{a} J^{\sigma} \chi^{a}-J^{\sigma}\right)\right|_{-\infty} ^{\infty}, E_{-\alpha}\right\rangle=\mathrm{i}\left[\left[e_{-\alpha}^{(a)}(x, \lambda), \operatorname{ad}_{J}^{-1}\left[J^{\sigma}, q\right]\right]\right] .
$$

Taking into account the construction of $\chi^{a}(x, \lambda)$ (see formulae (16), (17)) and the parametrization of the Gauss factors (18) one obtains

$$
\begin{aligned}
& {\left[\left[e_{-\alpha}^{(a)}(x, \lambda), \operatorname{ad}_{J}^{-1}\left[J^{\sigma}, q\right]\right]\right]=\mathrm{i} \alpha\left(J^{\sigma}\right) s_{a, \alpha}^{+}} \\
& {\left[\left[e_{\alpha}^{(a)}(x, \lambda), \operatorname{ad}_{J}^{-1}\left[J^{\sigma}, q\right]\right]\right]=-\mathrm{i} \alpha\left(J^{\sigma}\right) s_{a, \alpha}^{-} .}
\end{aligned}
$$


By analogy, the variation of $q$ can be expanded in the following manner $\operatorname{ad}_{J}^{-1} \delta q(x)=\frac{\mathrm{i}}{2 \pi} \sum_{a=1}^{6}(-1)^{a+1} \int_{l_{a}} \mathrm{~d} \lambda\left(\delta s_{a, \beta_{a}}^{+} e_{\beta_{a}, 1}^{(a)}(x, \lambda)-\delta s_{a, \beta_{a}}^{-} e_{-\beta_{a}, 1}^{(a-1)}(x, \lambda)\right)$.

The latter is obtained starting from another Wronskian relation

$$
\left.\hat{\chi}^{a} \delta \chi^{a}\right|_{-\infty} ^{\infty}=\mathrm{i} \int_{-\infty}^{\infty} \mathrm{d} x \hat{\chi}^{a} \delta q \chi^{a}
$$

Let us introduce the quantities

$$
\mathscr{E}_{\alpha}^{(a)}=\chi^{a} E_{\alpha} \hat{\chi}^{a}=e_{\alpha}^{(a)}+d_{\alpha}^{(a)}, \quad \mathscr{H}_{j}^{(a)}=\chi^{a} H_{j} \hat{\chi}^{a}=h_{j}^{(a)}+f_{j}^{(a)}
$$

to satisfy the equations

$$
\begin{aligned}
\mathrm{i} \partial_{x} \mathscr{E}_{\alpha}^{(a)}+\left[q-\lambda J, \mathscr{E}_{\alpha}^{(a)}\right] & =0 \\
\mathrm{i} \partial_{x} \mathscr{H}_{j}^{(a)}+\left[q-\lambda J, \mathscr{H}_{j}^{(a)}\right] & =0 .
\end{aligned}
$$

For the sake of simplicity we are going to skip the upper index $a$ since all further considerations do not depend on its choice. After splitting the diagonal and offdiagonal part of the above equations we get

$$
\begin{aligned}
\mathrm{i} \partial_{x} e_{\alpha}+\pi\left[q, e_{\alpha}\right]+\pi\left[q, d_{\alpha}\right] & =\lambda \pi\left[J, e_{\alpha}\right] \\
\mathrm{i} \partial_{x} d_{\alpha}+(\mathbb{1}-\pi)\left[q, e_{\alpha}\right] & =0 .
\end{aligned}
$$

By analogy with the very "squared" solution the diagonal parts of $\mathscr{E}_{\alpha}$ admit an expansion to be compatible with the grading

$$
d_{\alpha}=\mathrm{d}_{\alpha}^{1} J^{2}+\mathrm{d}_{\alpha}^{2} J
$$

Substituting it into (24) one immediately gets

$$
\mathrm{i} \partial_{x} \mathrm{~d}_{\alpha}^{\sigma}+\frac{1}{3} \operatorname{tr}\left(\left[q, e_{\alpha, \sigma}\right] J^{\sigma}\right)=0 \Rightarrow \mathrm{d}_{\alpha}^{\sigma}=\frac{\mathrm{i}}{3} \int_{ \pm \infty}^{x} \mathrm{~d} y \operatorname{tr}\left(\left[q, e_{\alpha}\right] J^{\sigma}\right), \quad \sigma=1,2 .
$$

On the other hand one can rewrite (23) by "components" as follows

$$
\begin{aligned}
\mathrm{i} \partial_{x} e_{\alpha, 0} & +\pi\left[q, e_{\alpha, 0}\right]=\lambda \pi\left[J, e_{\alpha, 1}\right] \\
\mathrm{i} \partial_{x} e_{\alpha, \sigma} & +\frac{\mathrm{i}}{3} \pi\left[q, J^{3-\sigma}\right] \int_{ \pm \infty}^{x} \mathrm{~d} y \operatorname{tr}\left(\left[q, e_{\alpha, \sigma}\right] J^{\sigma}\right)+\pi\left[q, e_{\alpha, \sigma}\right]=\lambda \pi\left[J, e_{\alpha, \sigma+1}\right] .
\end{aligned}
$$


As a result we obtain

$$
\Lambda_{0} e_{\alpha, 0}=\lambda e_{\alpha, 1}, \quad \Lambda_{\sigma} e_{\alpha, \sigma}=\lambda e_{\alpha, \sigma+1}
$$

where

$$
\begin{aligned}
& \Lambda_{0}=\operatorname{ad}_{J}^{-1}\left(\mathrm{i} \partial_{x}+\pi[q, .]\right) \\
& \Lambda_{\sigma}=\operatorname{ad}_{J}^{-1}\left\{\mathrm{i} \partial_{x}+\frac{\mathrm{i}}{3} \pi\left(\left[q, J^{3-\sigma}\right]\right) \int_{ \pm \infty}^{x} \mathrm{~d} y \operatorname{tr}\left([q, .] J^{\sigma}\right)+\pi[q, .]\right\} .
\end{aligned}
$$

Therefore we have

$$
\Lambda e_{\alpha}=\lambda^{3} e_{\alpha}, \quad \Lambda=\Lambda_{2} \Lambda_{1} \Lambda_{0} \oplus \Lambda_{0} \Lambda_{2} \Lambda_{1} \oplus \Lambda_{1} \Lambda_{0} \Lambda_{2}
$$

where each term in $\Lambda$ acts in a different subspace (from $\mathfrak{g}^{0}$ to $\mathfrak{g}^{2}$ ).

It can be verified that the integrable hierarchy of Kaup-Kupershmidt equation in terms of $\Lambda$ operator reads

$$
\begin{aligned}
\operatorname{iad}_{J}^{-1} \partial_{t} q & =\sum_{l=1}^{n} c_{3 l-1} \Lambda^{l-1} \Lambda_{0} \Lambda_{2} \operatorname{ad}_{J}^{-1}\left[q, J^{2}\right]-\sum_{l=1}^{n} c_{3 l-2} \Lambda^{l-1} \Lambda_{0} q, N=3 n-1 \\
\operatorname{iad}_{J}^{-1} \partial_{t} q & =\sum_{l=1}^{n-1} c_{3 l-1} \Lambda^{l-1} \Lambda_{0} \Lambda_{2} \operatorname{ad}_{J}^{-1}\left[q, J^{2}\right]-\sum_{l=1}^{n} c_{3 l-2} \Lambda^{l-1} \Lambda_{0} q, N=3 n-2
\end{aligned}
$$

where

$$
\mathbf{f}(\lambda)=\sum_{m=1}^{n}\left(c_{3 m-1} \lambda^{3 m-1} J^{2}+c_{3 m-2} \lambda^{3 m-2} J\right), \quad c_{3 m}=0 .
$$

In particular, for the Kaup-Kupershmidt equation itself we have $\mathbf{f}(\lambda)=-9 \lambda^{5} J^{2}$ and therefore

$$
\operatorname{iad}_{J}^{-1} \partial_{t} q+9 \Lambda \Lambda_{0} \Lambda_{2} \operatorname{ad}_{J}^{-1}\left[q, J^{2}\right]=0 .
$$

After substituting the expansions of $q$ and its variation one obtains

$\mathrm{i} \partial_{t} s_{a, \beta_{a}}^{ \pm} \mp 9 \lambda^{5} \beta_{a}\left(J^{2}\right) s_{a, \beta_{a}}^{ \pm}=0 \Rightarrow s_{a, \beta_{a}}^{ \pm}(t)=s_{a, \beta_{a}}^{ \pm}(0) \exp \left(\mp 9 \mathrm{i} \beta_{a}\left(J^{2}\right) \lambda^{5} t\right)$.

In the general case we have

$$
s_{a, \beta_{a}}^{ \pm}(t)=\exp \left[ \pm \mathrm{i}\left(\beta_{a}\left(J^{2}\right) \sum_{l} c_{3 l-1} \lambda^{l+1}+\beta_{a}(J) \sum_{l} c_{3 l-2} \lambda^{l}\right) t\right] s_{a, \beta_{a}}^{ \pm}(0) .
$$

These are linearized versions of the NEE with dispersion laws $\mathbf{f}(\lambda)$. 


\section{Conclusion}

We have realized the generalized Fourier transform interpretation of the ISM in the case of Kaup-Kupershmidt type equations in terms of "squared solutions" and recursion operators. The presence of a $\mathbb{Z}_{3}$ symmetry leads to a $\mathbb{Z}_{3}$-grading in $\mathfrak{s l}(3, \mathbb{C})$. As a result the completeness property of the "squared solutions" holds true also for their components. Due to compatibility requirements with the $\mathbb{Z}_{3^{-}}$ grading the $\Lambda$-operator obtains a certain factorization. Thus one can describe the integrable hierarchy of the KKE by using the $\Lambda$-operator and its factors. Moreover, by using expansions over the "squared solutions" one is able to obtain linearized versions of the corresponding NEE in terms of minimal sets of scattering data. Apart from describing the hierarchy of integrable NEE the $\Lambda$-operators generate an infinite hierarchy of symplectic forms and Hamiltonians associated with these equations. Discussed results can be generalized for Lax operators related to $\mathfrak{s l}(r+1)$ and even related to an arbitrary simple Lie algebra. For example, the "squared solutions" admit the following expansion

$$
e_{\alpha}(x, \lambda)=\sum_{k=0}^{h-1} e_{\alpha, k}(x, \lambda), \quad e_{\alpha, k}(x, \lambda) \in \mathfrak{g}^{k}
$$

where $h$ is the Coxeter number of the Lie algebra $\mathfrak{g}$. The corresponding recursion operator can be represented

$$
\Lambda=\Lambda_{h-1} \Lambda_{h-2} \ldots \Lambda_{0}+\text { cycl. }
$$

where

$$
\Lambda_{k_{j}}=\operatorname{ad}_{J}^{-1}\left(\mathrm{i} \partial_{x}+\pi[q, .]+\mathrm{i} \pi\left[q, \mathcal{E}_{j}\right] \int_{ \pm \infty}^{x} \mathrm{~d} y\left\langle[q, .], \tilde{\mathcal{E}}_{j}\right\rangle\right)
$$

if $\mathfrak{g}^{k_{j}}$ intersects the centralizer of $J$ and

$$
\Lambda_{k_{j}}=\operatorname{ad}_{J}^{-1}\left(\mathrm{i} \partial_{x}+\pi[q, .]\right)
$$

otherwise. The elements $\mathcal{E}_{j} \in \mathfrak{g}^{k_{j}}$ and $\tilde{\mathcal{E}}_{m} \in \mathfrak{g}^{l_{m}}$ form a basis in the centralizer of $J$ and they are normalized as follows

$$
\left\langle\mathcal{E}_{j}, \tilde{\mathcal{E}}_{m}\right\rangle=\delta_{j m}
$$

In the case of $\mathfrak{s l}(r+1)$ these are simply certain powers of $J$. Another possible generalization is to consider NEEs with constant boundary conditions. This class of NEEs currently is a subject of increasing interest, see [8]. 


\section{Acknowledgements}

The author acknowledges support from the European Operational programm HRD, contract BGO051PO001/07/3.3-02/53 with the Bulgarian Ministry of Education. The author would like to thank also to Professor Vladimir Gerdjikov for useful discussions.

\section{References}

[1] Beals R. and Coifman R., Scattering and Inverse Scattering for First Order Systems, Commun. Pure Appl. Math. 37 (1984) 39-90.

[2] Beals R. and Coifman R., Scattering and Inverse Scattering for First Order Systems II, Inv. Problems 3 (1987) 577-593;

Beals R. and Coifman R., Linear Spectral Problems, Nonlinear Equations and the Delta-Method, Inv. Problems 5 (1989) 87-130.

[3] Caudrey P., The Inverse Problem for a General $N \times N$ Spectral Equation, Physica D 6 (1982) 51-66.

[4] Drinfel'd V. and Sokolov V., Lie Algebras and Equations of Korteweg-de Vries Type, Sov. J. Math. 30 (1985) 1975-2036.

[5] Fordy A. and Gibbons J., Factorization of Operators I. Miura Transformation, J. Math. Phys. 21 (1980) 2508-2510.

[6] Gerdjikov V., Generalised Fourier Transforms for the Soliton Equations. Gauge-Covariant Formulation, Inv. Problems 2 (1986) 51-74.

[7] Gerdjikov V. and Yanovski A., Completeness of the Eigenfunctions for the Caudrey-Beals-Coifman System, J. Math. Phys. 35 (1994) 3687-3725.

[8] Gerdjikov V. and Kostov N., Multi-component Nonlinear Schrödinger Equation on Symmetric Spaces with Constant Boundary Conditions. Part I, JGSP 19 (2010) 1-28.

[9] Grahovski G., On the Reductions and Scattering Data of CBC System, In: Geometry, Integrability and Quantization II, I. Mladenov and G. Naber (Eds), Coral Press, Sofia 2001, pp 262-277.

[10] Humphreys J., Introduction to Lie Algebras and Representation Theory, Springer, New York, 1978.

[11] Kaup D., On the Inverse Scattering Problem for Cubic Eigenvalue Problems of the Class $\psi_{x x x}+6 Q \psi_{x}+6 R \psi=\lambda \psi$, Stud. Appl. Math. 62 (1980) 189216. 
[12] Mikhailov A., The Reduction Problem and The Inverse Scattering Method, Physica D 3 (1981) 73-117.

[13] Takhtadjan L. and Faddeev L., The Hamiltonian Approach to Soliton Theory, Springer, Berlin, 1987.

[14] Valchev T., On the Kaup-Kupershmidt Equation. Completeness Relations for the Squared Solutions, In: Geometry, Integrability and Quantization IX, I. Mladenov (Ed), Softex, Sofia 2008, pp 308-319.

[15] Zakharov V., Manakov S., Novikov S. and Pitaevskii L., Theory of Solitons: The Inverse Scattering Method, Plenum, New York, 1984.

Tihomir Valchev

Institute for Nuclear Research

and Nuclear Energy

Bulgarian Academy of Sciences

1784 Sofia, BULGARIA

E-mail address: valtchev@inrne.bas.bg 